Cardiology 1997;88:619-620

\title{
Subject Index Vol. 88,1997
}

Accessory atrioventricular pathway, posteroseptal 328

ACE inhibition 285

Acetylcholine 503

Acute cardiogenic pulmonary edema 509

coronary syndromes 223

myocardial infarction 223, 482,544

Adenosine 180 ß-Adrenergic receptor 418 Adult, case report 478 Aging 147 Aldosterone 6 [13N]Ammonia 106 Anabolic-androgenic steroids

218 Angiography 530 Angioplasty 533 Angiotensin II 6 Angiotensin-convertmg enzyme

309 Antegrade cardioplegia 526 Antihypertensive therapy 277 Aortic regurgitation 418

- $\quad$ stenosis 315

valve replacement 315 Arrhythmias 141 Arterial hypertension 277

pulses 408 Atrial fibrillation 563

natriuretic peptide 6,238

pacing 576

septal aneurysm 300

- defect 609

tachycardia 474 Atrioventricular block 474,

613 Auscultatory sites 408 Automated border detection

393 Automatic mode switch 323 AV node ablation 323 AV-synchronous pacing 323

Balloon angioplasty 433

anulus ratio 271

dilatation 271 Behçet's disease 513 Betablocker 478 Bland-White-Garland

syndrome 603

ß-Blocker 418

Body position 576

Brain natriuretic peptide 401

Calcium antagonist 353

- $\quad$ channel blocker(s) 292, 478

Cardiac DDD(R) pacemaker

323

hypertrophy 518

impulses 408

output 19

pacemaker 478

pacing 264

rehabilitation 207, 595

tamponade 48, 397 Cardiogenic shock 482 Cardiology patient simulator 
408 Cardiomyopathy 189 Cardiopulmonary indices 595 Cardiotonic agents 292

Cardiovascular toxicity 218 Carnitine palmitolytransferase

258 Carotid phonoarteriography

147 Catecholamine 418 Catheter ablation 346 Causal attribution 29, 367 Cholesterol 503

Chronic salt restriction 156 Circadian rhythm 509 Clinical ward 116 Community study 460

Complete remission 613 Complications 530 Congestive heart failure 585 Contractile function

14 Contrast angiography 116

- $\quad$ fluid 576

Coping 29, 367

Coronary arteries, endothelium-dependent dilation 414

artery 603

- bypass graft 425

- disease 180,246,258,

353,379,533,556,595

- grafting 333

- surgery 492

blood flow 80,433

bypass surgery 207

care 223

- unit 373

circulation 1

flow reserve $116,425,433$

lesion severity 386

restenosis 433

stent 530

vasospasm 503 Coxsackie virus 296 CTscan 264

Diabetes mellitus 152 Diagnostic imaging 292 Diastolic function 36, 203 Digital and

computer technology 408 Dilated cardiomyopathy 156,

218,258,401 Dipyridamole 569 Divisional block 127 Dobutamine 180

stress echocardiography 197,379,386,556

thallium-201 scintigraphy 556

Doppler echocardiography 203,

498 Dynamic emission data 54

ECG changes, long-term

outcome, prognosis 460 Echocardiogram 254 Echocardiography 26, 170, 189,

361 -, transesophageal 292 Eisenmenger reaction 609 Elderly patients 401 Electrocardiogram

361,533 Electrocardiography 328 Electroconvulsive therapy 254 Electromechanical

dissociation

478 Enalapril 6

Endoaneurysmorrhaphy 340 Endothelin 1 Endothelium 425 Essential hypertension 141, 238,277,309,401 Exercise capacity 6,207 -, QRS duration 446

test $36,238,246$

testing 207, 453, 595

thallium-201 SPECT 379 
- $\quad$ training 468, 595

Experimental model 518

Factor V deficiency 48 False-positive results 453 Familial dysautonomia 133 Fick 19

Fluvastatin 160

Gallopamil 353 Gene polymorphism 309 Gensini’s score 386 Giant cell 296 Goiter 156

Handgrip-apexcardiographic

test 36 Heart disease 513

failure 246,418

injuries 292

rate variability 133 Hemiblock 127 Hemodynamics 478, 548 Hexaxial system 127

Hibernation 333 Human immunodeficiency

virus 397

- $\quad$ - - infection 26

3-Hydroxy-3-methylglutaryl

coenzyme A reductase

inhibitors 160 Hyperlipidemia 160 Hypertension 36, 147 -, redevelopment 277 Hypertrophy

189 Hypotension 285,478

Inferior wall acute myocardial

infarction 361 Insulin 503 Interventricular septal wall

motion 526 Intracoronary Doppler 433 Ischemia 446, 533 Ischemic heart disease 62, 453, 540

Japanese women 309 Jugular-venous waves 408

Kinetic modeling and analysis 54

KARGER

E-Mail karger@karger.ch Fax + 41613061234 http://www. karger. ch

(C)1997 S.KargerAG, Basel

619

Laser 346

Left anterior descending artery 197

atrial appendage 563

bundle branch 127

circumflex artery 197

main occlusion 482

ventricular aneurysm 340

- diastolic function 498

- dysfunction 26,315,

$397,595,601$

- filling 203

- function 152,246,254,

393

- hypertrophy 309

- mass 468

- volume 393 Logistic model 453 Long-term survival 333 Lung cancer 214

Magnetic resonance 80 Marathon runners 487 Mechanocardiogram 408 Media model 90

Microcirculation 425 Mild hypertension 468 Mitochondria 258 Mitral balloon valvotomy 300 
echogram 373

valve prolapse 513 Monitoring 576 Morphological changes 315 MRI contrast 90 Myocardial blood flow 71,106,

116

- -, flow reserve 62

- - heterogeneity 62

infarction 29,166,203, 207,285,367,373

-, location 441

-, ramipril 548

ischemia 14,141,285,569, 576

- $\quad$ perfusion 353

Myocarditis 296

Myocardium 90

Myotonic dystrophy 498

Necrosis 333 Neurohormones 548 Nitroglycerin 170 Normal coronary arteries 453

Orthostatic hypotension 133 Osteosarcoma 214 Outcome 29 -, subjective and objective

367 Oxygen consumption 19

Paroxysmal atrial fibrillation/

flutter 323 Patent foramen ovale 300 Perfusion 90

Pericardial effusion 397, 544 Pericardiectomy 48 Perioperative infarction 333 pH 14

Phenylephrine 292 Physiology laboratory 116 Plasma catecholamines 238

- $\quad$ fibrinogen 468

Positron emission tomography

54,62,106

Precordial ST-segment depression 361

Predictors, hypertension 277

Primary cardiac lymphoma 613

Prior myocardial infarction 401

Probucol 160

Prognosis 492

Prognostic significance, infarction location 441

Protein synthesis 518

Proteolysis 518

Pulmonary artery catheter 478

capillary wedge pressure 373

hypertension 609

valve 271

venous flow 214

- - velocity 585 Pulse transmission time 147

wave velocity 147

QRS scoring 333 QT intervals 133 Quantitative wall motion analysis 386

Rabbit 518 Radiofrequency 328, 346

ablation 328 Radionuclide(s) 71

ventriculography 152, 180 Ramipril, tolerability 548 Recurrent infarction, infarction 
location 441 Rehabilitation 29 Remodeling 170 Renal blood flow 540 Renin 6 Reninangiotensin-aldosterone

system 238 Reperfusion 170,223 Rescue percutaneous translu-

minal coronary angioplasty

482 Resuscitation 292 Retrograde cardioplegia 526 Right coronary artery 197

- $\quad$ ventricular sympathetic

denervation 609

Risk assessment 569 Rubidium-82 54

Segmental wall motion 361

Sepsis 19

Serum cardiac markers (CK-

$\mathrm{MB}$, cardiac troponin $\mathrm{T}$,

cardiac troponin I) 487 Shock, cardiogenic 292 Sick sinus syndrome 264 Signal-averaged

electrocardiog-

raphy 133 Single origin 603 Sinus node dysfunction 166 Smoking 492 Spongy myocardium

601 ST segment depression 460 Stroke 264 Structural heart disease,

absence 474

Stunned myocardium 487 Stunning 170 Surgery 569 Syndrome X 414

Thallium 569

Thallium-201 myocardial scin-tigraphy 353

Thermodilution 19

Three-dimensional reconstruction 54

Thrombolysis 544

Thyroid disorders 156

Tracers 71

Transesophageal echocardiog-raphy 214,300,585

Tuberculous pericarditis 397

Two-dimensional reconstruction 54

Ultrasound 530 Unstable angina 223 Urinary oxygen tension 540

Valvular heart disease 401, 518 Vasomotion 414 Vectorcardiography 576 Ventricular

diastole 563

function 292

outflow obstruction 292

tachycardia 346 Verapamil toxicity 478 Volume of interest 54

overload 518

WHO Monica project 460 Zidovudine 26

620

Cardiology Vol. 88, 1997

Subject Index 